\title{
Disease gene identification strategies for exome sequencing
}

\author{
Christian Gilissen $^{\star, 1}$, Alexander Hoischen ${ }^{1}$, Han G Brunner ${ }^{1}$ and Joris A Veltman ${ }^{1}$
}

Next generation sequencing can be used to search for Mendelian disease genes in an unbiased manner by sequencing the entire protein-coding sequence, known as the exome, or even the entire human genome. Identifying the pathogenic mutation amongst thousands to millions of genomic variants is a major challenge, and novel variant prioritization strategies are required. The choice of these strategies depends on the availability of well-phenotyped patients and family members, the mode of inheritance, the severity of the disease and its population frequency. In this review, we discuss the current strategies for Mendelian disease gene identification by exome resequencing. We conclude that exome strategies are successful and identify new Mendelian disease genes in approximately $60 \%$ of the projects. Improvements in bioinformatics as well as in sequencing technology will likely increase the success rate even further. Exome sequencing is likely to become the most commonly used tool for Mendelian disease gene identification for the coming years.

European Journal of Human Genetics (2012) 20, 490-497; doi:10.1038/ejhg.2011.258; published online 18 January 2012

Keywords: Mendelian disease; gene identification; strategies; next generation sequencing; exome sequencing

\section{INTRODUCTION}

The number of rare monogenic diseases is estimated to be $>5000$ and for half of these the underlying genes are unknown. ${ }^{1}$ In addition, an increasing proportion of common diseases, such as intellectual disability, schizophrenia, and autism, previously thought to be due to complex multifactorial inheritance, are now thought to represent a heterogeneous collection of rare monogenic disorders, ${ }^{2-5}$ the large majority of which is still unknown. The identification of genes responsible for these diseases enables molecular diagnosis of patients, as well as testing gene carriers and prenatal testing. Gene identification represents the first step to a better understanding of the physiological role of the underlying protein and disease pathways, which in turn serves as a starting point for developing therapeutic interventions. ${ }^{6}$ Recent advances in next generation sequencing technologies have dramatically changed the process of disease gene identification, in particular by using exome sequencing in which the protein-coding part of the genome of a patient can be studied in a single experiment (see Majewski et $a l^{7}$ for an overview of exome sequencing technology and its applications). As tens of thousands of genomic variants can be identified in each exome, it is important to carefully consider strategies for efficiently and robustly prioritizing pathogenic variants. In order to do so, much can be learned from traditional disease gene identification approaches, but also novel strategies need to be established.

\section{TRADITIONAL DISEASE GENE IDENTIFICATION}

Past identification of Mendelian disease genes was carried out by Sanger sequencing of candidate genes. Candidate genes can be selected because they resemble genes associated with similar diseases, because the predicted protein function seems relevant to the physiology of the disease, or because a positional mapping approach pointed to these genes in a genomic region. ${ }^{8}$ This last approach has been most successful as it does not rely on prior biological or medical knowledge and can be applied in an unbiased fashion. The most important genetic mapping approaches rely on karyotyping, ${ }^{9}$ linkage analysis, ${ }^{10}$ homozygosity mapping, ${ }^{11}$ copy number variation analysis, ${ }^{12}$ and SNPbased association analysis. ${ }^{13}$ One problem associated with using genetic mapping approaches is that it is difficult, if not impossible, to predict whether a disease is caused by a single nucleotide mutation or by structural genomic variation. Without family information it is also often difficult to predict whether a disease is dominantly or recessively inherited. Therefore, different mapping approaches often need to be applied in a sequential order before a disease locus is identified. In addition, these mapping approaches commonly do not reduce the number of candidate genes sufficiently for follow-up by Sanger sequencing, when the disease locus remains very large. This is especially the case if these mapping approaches are applied to only a single patient or family with a limited number of informative relatives. Combining data from multiple unrelated but phenotypically similar patients or families is useful to reduce this to a manageable number, but carries a risk that patients with similar phenotypes are affected by mutations in different genes. Alternatively, a candidate gene approach can be used to select the best candidate genes from the large disease locus for Sanger sequencing. Many bioinformatics tools have been developed to prioritize candidate disease genes from disease gene loci. ${ }^{14-16}$ Candidate gene selection is however critically dependent on prior knowledge and only few disease genes have been identified by specifically using these bioinformatics tools. ${ }^{17} \mathrm{~A}$ particular category of diseases that has remained largely unresolved is that of the rare genetic disorders that occur sporadically, and for which neither a family-based approach nor an association-based approach can be used. These

${ }^{1}$ Department of Human Genetics, Nijmegen Centre for Molecular Life Sciences and Institute for Genetic and Metabolic Disorders, Radboud University Nijmegen Medical Centre, Nijmegen, The Netherlands

${ }^{*}$ Correspondence: C Gilissen, Department of Human Genetics, Nijmegen Centre for Molecular Life Sciences and Institute for Genetic and Metabolic Disorders, Radboud University Nijmegen Medical Centre, 6500 HB Nijmegen, The Netherlands. Tel: +31243668940; E-mail: c.gilissen@antrg.umcn.nl

Received 24 August 2011; revised 31 October 2011; accepted 7 December 2011; published online 18 January 2012 
diseases require unbiased gene identification approaches that can be successfully applied in small cohorts or single patients.

\section{NEXT GENERATION SEQUENCING (NGS) TECHNOLOGY}

NGS technology is changing medical genomics by making highthroughput sequencing of DNA and RNA available and affordable. ${ }^{18}$ Mendelian disease gene identification in particular benefits from this development. Rapid sequencing of the entire genome of a patient removes the necessity to prioritize candidate genes for sequencing, and can therefore reduce the disease gene identification process from a two-step approach (positional mapping followed by Sanger sequencing) to a one-step approach (whole genome sequencing). The technical simplicity of this approach allows large-scale application in Mendelian disease research and diagnostics. With NGS, the disease gene identification challenge shifts from the identification to the interpretation phase; millions of genomic variants are identified per genome but only one or two may explain the Mendelian disease. Prioritization of variants is therefore, crucial to the disease gene identification process. For (severe) Mendelian disorders prioritization assumes that the mutation has a large effect, and is therefore (1) unique in patients or at least very rare in the general population, (2) located within the protein-coding regions of the genome, and (3) directly affecting the function of the protein encoded by the mutated gene. ${ }^{19}$

Most whole genome studies published so far have focused on the $2 \%$ of the genome that is coding, as roughly $85 \%$ of the known genetic causes for Mendelian disorders affect the protein coding regions (although this may have to do with an ascertainment bias). ${ }^{8}$ This reduces the amount of variants from 3 to 4 million to $<25000$ for follow-up. ${ }^{20-24}$ Because whole genome sequencing is still limited in throughput and too costly to be applied as the main tool for disease gene discovery, different capturing approaches have been developed to enrich the exome before NGS. ${ }^{25}$ The advantage of this enrichment is that many more exomes than genomes can be sequenced per NGS system per run, and despite the additional enrichment, costs are lower by a factor of 5-10. A single run on the latest generation of such NGS systems can generate enough sequence data to simultaneously study up to 100 exomes in parallel.

\section{Disease gene identification strategies for exome sequencing}

The number of variants that are identified in exome sequencing studies varies greatly. This depends on the exome enrichment set that was used, the sequencing platform and the algorithms used for mapping, and variant calling (see Figure 1). Typically, between 20000 and 50000 variants are identified per sequenced exome. In order to reduce the number of false-positive calls, variants are first filtered based on quality criteria, such as the total number of independent reads showing the variant (eg, at least five independent reads), and the percentage of reads showing the variant (eg, at least 20\% for heterozygous variants, at least $80 \%$ for homozygous variants). Subsequently, variants outside the coding regions can be filtered out, as well as synonymous coding variants, on the basis of the assumption that these will have minimal effect on the protein, as described above. This reduces the number of potential disease-causing variants to roughly 5000. The most substantial reduction follows from excluding known variants (commonly from dbSNP, published studies, ${ }^{26,27}$ or in-house databases). This step typically reduces the number of potential candidate mutations by $90-95 \%$. After this, typically between 150 and 500 private non-synonymous or splicesite variants are prioritized as potential pathogenic variants (see Figures 1 and 2). ${ }^{4,28-34}$
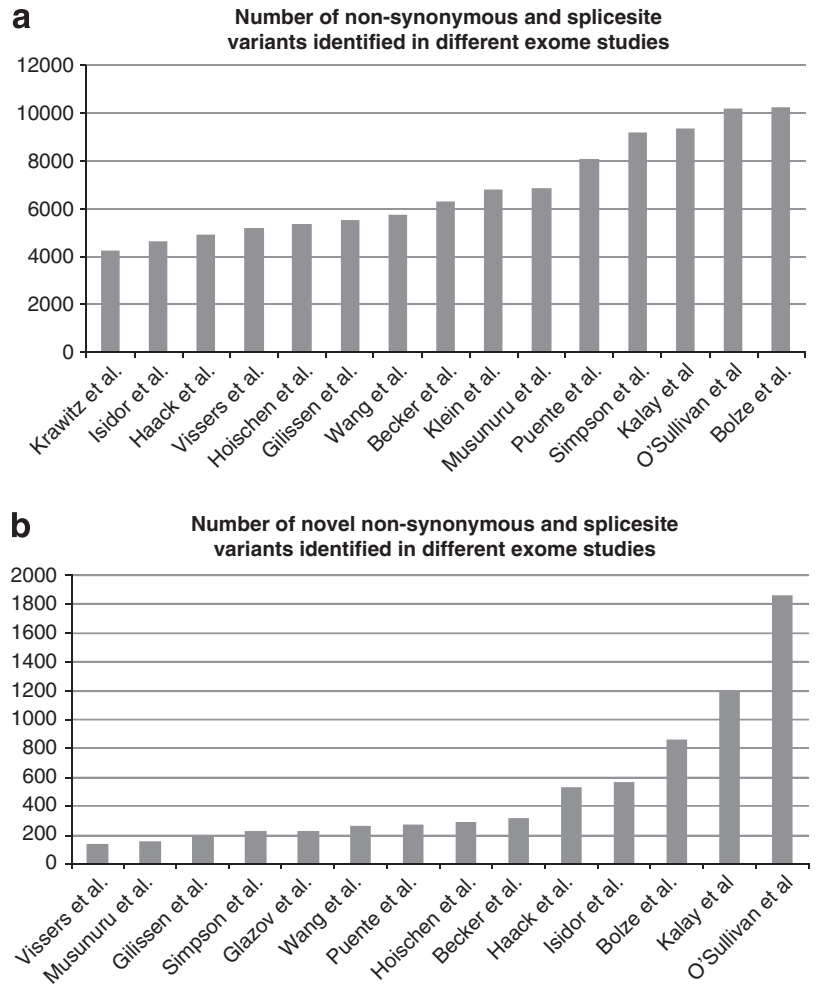

Figure 1 Number of variants identified in published exome studies. (a) Number of non-synonymous variants identified in published exome studies. From left to right: ${ }^{36,73,80,74,33,32,51,31,82,52,83,72,84,85,61}$. (b) Number of novel non-synonymous variants identified in published exome studies. From left to right: ${ }^{74,52,32,72,57,51,83,33,31,80,73,61,85,84}$.

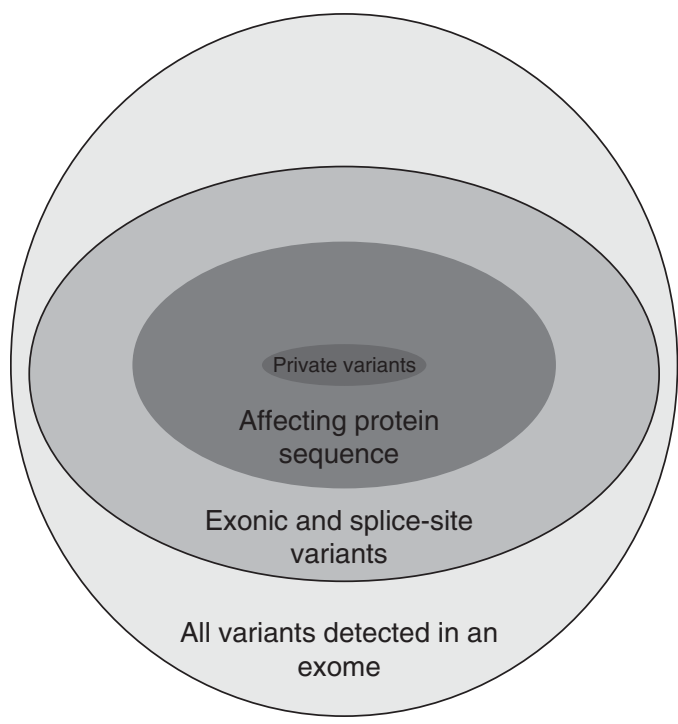

Figure 2 Prioritization of NGS variants. Common prioritization of variants. The size of the enclosing ellipses is indicative of the relative number of variants that remain after each prioritization step.

It is important to emphasize that prioritization may discard the pathogenic variant. A variant that is present at low frequency in a heterozygous state in the normal population may be removed even though it causes disease if present in a homozygous state. For a very 
a

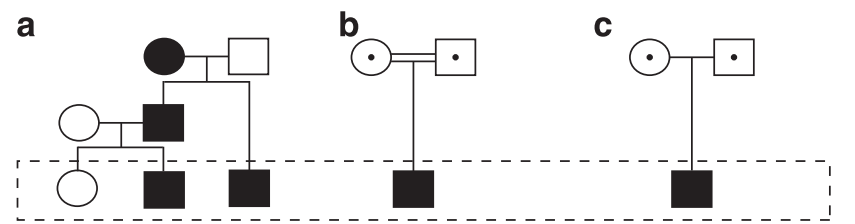

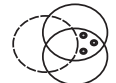

Linkage based strategy

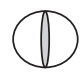

Homozygosity based strategy

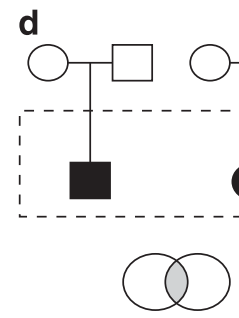

Overlap based strategy e
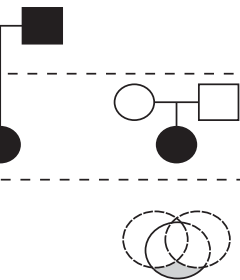

De novo based strategy

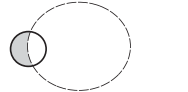

Double-hit based strategy

f
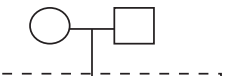

Figure 3 Disease gene identification strategies for exome sequencing. The strategies (a-f) are detailed in the main text. Pedigrees indicate the inheritance model loosely underlying the strategy; filled symbols represent affected individuals, empty symbols represent presumably healthy individuals, and carriers are depicted by a symbol with a dot. Dashed rectangle encloses individuals that are exome sequenced. Circles below each pedigree symbolize sets of genetic variants identified in the exomes. Solid circles represent variants from affected individuals, whereas dashed circles represent variants from unaffected.

rare recessive disorder, the expected carrier frequency in the population will be extremely low, which allows for the use of large control sets to exclude previously identified variants. To assume that there are no carriers for recessive disorders in the population and thereby in the control data is dangerous, especially when the disease occurs more frequently. Hence, it is important that reliable population frequencies are obtained for variants in control data sets, as this will allow the exclusion of variants that are present at frequencies higher than the expected carrier frequency. Walsh et $a l^{35}$ warned about the use of uncurated variant databases. One of the pathogenic mutations they identified was recorded in dbSNP as a known variant based on a single study. The authors did not observe the variant in 768 individuals, which led them to speculate that the entry was likely an artifact due to alignment errors in short mononucleotide runs.

It is clear that this initial prioritization is no more than a first selection, and will rarely identify the pathogenic variant by itself. Additional strategies are needed to find the causative mutation among this list of $150-500$ private variants. For this, both traditional positional mapping strategies as well as other common approaches have been adapted for exome sequencing (Figure 3, Table 1). We briefly discuss each of these strategies:

Linkage strategy. For a family with a monogenic inherited disorder, multiple affected family members can be sequenced to identify shared variation. In addition, non-affected family members can be sequenced to exclude private benign variation. By selecting the most distantly related affected family members, the amount of shared benign variation can be kept to a minimum. For example, two affected siblings are expected to share about $50 \%$ of their DNA. By combining sequence data, this will lead to a similar reduction in private variants that needs

to be considered. The first application of this approach was by $\mathrm{Ng}$ et a ${ }^{29}$ who used data from two affected siblings to determine shared variation, reducing the amount of recessive candidates to a mere 9 genes. Krawitz et $a l^{36}$ applied a more sophisticated version of this approach using only exome sequencing data. The authors determined the haplotype for all variants shared by three affected siblings, and selected those variants that are identical by descent. In this way they reduced the number of candidate genes from 14 to only 2 genes.

Homozygosity strategy. In case of a rare recessively inherited disorder and suspected consanguinity, the initial assumptions are that the disease is caused by a homozygous variant inherited from both parents and that this variant resides within a large stretch of a homozygous region. Homozygous variants can therefore be prioritized by their presence in large homozygous regions of the patient's genome. These regions can be identified by SNP microarrays and used during the prioritization process, ${ }^{35}$ but Becker et al ${ }^{31}$ recently showed that exome data itself may contain sufficient numbers of informative SNPs to allow reliable homozygosity mapping. In this study, 17 of the 318 private non-synonymous variants observed in the index patient were autosomal homozygous variants, but only three were located in large homozygous regions and the causative mutation was located in the largest of these three. The main difference of this strategy compared with the linkage strategy is that variants, although homozygous, are only selected when contained within a large homozygous stretch. As such, this approach reduces the amount of variants for follow-up sufficiently to allow disease gene identification in individual cases, and does not require additional family members, at least not for initial variant identification. This approach however, can only detect homozygous loci in regions with a sufficient target density containing informative SNPs.

Double-hit strategy. When only a single patient is available without additional family members and the disorder is suspected to be recessively inherited (but without any indication for consanguinity), it is possible to sequence only this single patient's exome and select for genes carrying homozygous as well as compound heterozygous variants, as there are relatively few of these private non-synonymous variants in the average outbred individual. Gilissen $e t a l^{32}$ used this strategy to reduce the number of candidate disease genes in two individuals with Sensenbrenner syndrome from 139 and 158 to only 3 and 4 , respectively. Pierce $e t a l^{37}$ used the same approach for a patient with Perrault syndrome, prioritizing a single gene among 207 private non-synonymous variants. Both studies show that this is a powerful approach that can identify the genetic cause of a disorder from just sequencing a single individual.

Overlap strategy. In the absence of genetic heterogeneity, one can search for mutations in a single gene in multiple unrelated patients with a similar phenotype. This is particularly important for disease gene identification in dominant disorders, as there are many more genes with private heterozygous non-synonymous variants than there are genes with private homozygous or compound heterozygote nonsynonymous variants. The number of genes with mutations in multiple affected patients will decrease rapidly by combining data from increasing numbers of patients, resulting in less candidate genes for follow-up. The first study to find the mutated gene for a dominant disorder by using this overlap approach in exome sequencing data used four unrelated individuals. ${ }^{33}$ Recently, we showed that combining data from three individuals was sufficient to identify the gene for Bohring-Opitz syndrome. ${ }^{34}$ Although in the individual patients we found between 130 and 222 novel non-synonymous variants, the 
Table 1 Overview of applicability, assumptions, advantages, and disadvantages for exome sequencing strategies

\begin{tabular}{lllll}
\hline Strategy & Applicability & Assumptions & Advantages & Disadvantages \\
\hline $\begin{array}{llll}\text { Linkage } \\
\text { (Figure 3a) }\end{array}$ & Multiple affected & Fully penetrant & Additional individuals can & Might require large \\
& within a single & mutation segregating & be sequenced to limit the & sequencing efforts, \\
& family. & with the disorder. & search space further. & affected share a lot \\
& & & of private variants.
\end{tabular}

$\begin{array}{lll}\text { Homozygosity } & \text { Single affected } & \text { Homozygous mutation } \\ \text { (Figure 3b) } & \text { from consanguine } & \text { within a homozygous } \\ & \text { parents. } & \text { stretch. }\end{array}$
parents.

\author{
Only a single patient \\ is required. \\ Only a single experiment \\ is required.
}

Relies on accuracy of sequencing technology.

\section{is required.}

Does not necessarily depend

on control data or other prioritization assumptions. Only a single patient is required.

Only a single experiment is required.

Does not necessarily depend on control data or other prioritization assumptions.

on current biological

knowledge.
The disorder might not necessarily be caused by a mutation in a homozygous region. Homozygosity based on exome data might suffer from limited resolution.

Relies on the availability of (ethnically matched) control data.

Depends on expected carrier frequency. Relies heavily on accurate assumption of a single involved locus. phenotyping and the

Examples

Krawitz et al ${ }^{36}$

Johnson et a/ ${ }^{54}$

Zuchner et al ${ }^{55}$

Norton et $a^{38}$

Wang et al ${ }^{51 a}$

Musunuru et al ${ }^{52 b}$

Yamaguchi et al ${ }^{56}$

Glazov et a/ ${ }^{57}$

Puente et al ${ }^{58}$

Gunay-Aygun et a/ ${ }^{59}$

Weedon et a/60

Becker et al ${ }^{31}$

Walsh et a/ ${ }^{35}$

Wang et al ${ }^{51 a}$

Bolze et $a l^{61}$

Caliskan et a/62

Bilguvar et a/63

O'Sullivan et al ${ }^{64}$

Barak et a/ ${ }^{65}$

Hanson et al ${ }^{66}$

Shaheen et al ${ }^{67}$

Doi et el 68

Gilissen et $a /^{32}$

Pierce et $a^{37}$

Musunuru et $a l^{52 b}$

Götz et al ${ }^{69 c}$

Murdock et al ${ }^{70}$

$\mathrm{Ng}$ et $\mathrm{al}^{71}$

Hoischen et a/ ${ }^{33}$

$\mathrm{Ng}$ et $\mathrm{al}{ }^{30}$

Simpson et al ${ }^{72}$

Isidor et al ${ }^{73}$

Vissers et al ${ }^{74}$

Albers et al ${ }^{75}$

Dickinson et al ${ }^{76}$

Sirmaci et al ${ }^{77}$

Agrawal et al ${ }^{78}$

Vissers et $a l^{4}$

O'Roak et al ${ }^{39}$

Xu et al ${ }^{5}$

Biased approach, relying

Byun et $\mathrm{al}^{79}$

Haack et al ${ }^{80}$

Worthey et $\mathrm{al}^{44}$

Götz et al ${ }^{69 c}$

Erlich et al ${ }^{45}$

Ozgul et al ${ }^{81}$

$\overline{a, b, c}$ Indicate studies that use a combination of two strategies.

overlap for any two patients yielded 26 candidate genes whereas the overlap from three patients pointed only to a single gene.

The overlap strategy will work best for true monogenic disease and will have to be adapted for diseases that show genetic heterogeneity. In addition, the incompleteness (ie, failure to enrich specific targets) of current exome sequencing data may cause a problem because pathogenic mutations may be missed in one or more patients, complicating the filtering strategy. A good example of both levels of complexity is displayed in the recent publication by $\mathrm{Ng}$ et $a l^{30}$ in which the authors sequenced the exomes of 10 patients with the clinical diagnosis of Kabuki syndrome. After filtering against existing SNP databases, no compelling candidate gene was identified that contained previously unknown variants in all affected individuals. Next, the authors conducted a less stringent analysis by looking for variation in candidate genes shared among subsets of affected individuals. This analysis pinpointed to $M L L 2$ as the major cause of this syndrome. 
On the basis of exome data, mutations in this gene were observed in 7 out of 10 patients. Sanger sequencing of the entire coding region of this gene in the mutation-negative cases, however, revealed MLL2 mutation in two out of the three cases, both being frameshift indels that were missed by exome sequencing. Similarly, one should be mindful of copy number variations, inversions, or translocations overlapping with the nucleotide variants identified in patients with the same phenotype, as these can cause the same disease but are easily missed by exome sequencing as illustrated by Norton et $a l^{38}$ The clinical and genetic heterogeneity of Kabuki syndrome was further highlighted in the replication cohort used in the study of $\mathrm{Ng}$ et al ${ }^{30}$ in which only $60 \%$ (26 out of 43 patients) of cases were found to have MLL2 mutations. The difference in the percentage of MLL2 mutationpositive cases between the discovery and replication cohort illustrates that the authors were able to clinically select canonical Kabuki cases for exome sequencing, highlighting the importance of accurate and consistent clinical phenotyping for successful disease gene identification using this overlap strategy.

De novo strategy. The overlap strategy described above only works for rare diseases that are largely monogenic. For common disorders that are genetically highly heterogeneous, the mutational target, that is, the amount of genome occupied by genes that when mutated result in disease, is too large to have a reasonable chance of finding two patients with mutations in the same gene. This large mutational target however, also increases the chance that a de novo mutation during meiosis occurs in one of these genes and causes disease. Especially when a disorder occurs mostly sporadic and is associated with reduced fecundity, as is the case for example for intellectual disability, the underlying cause can be hypothesized to lie with these de novo mutations. De novo mutations in these patients can be identified by using a family-based exome sequencing approach. By sequencing the exome of the patient as well as his or her parents, de novo candidates can be selected by filtering out all inherited variants. This will yield a limited number of potential pathogenic variants, as the average exome contains only $0-3$ de novo mutations. ${ }^{4,22,26,39}$ Vissers et al ${ }^{4}$ showed that de novo point mutations could be linked to disease in 7 out of 10 patients with intellectual disability, indicating that these mutations may explain the large majority of the genetic burden. These remarkable findings in intellectual disability have recently been replicated in sporadic forms of autism ${ }^{39}$ and schizophrenia, ${ }^{5}$ indicating that de novo mutations may explain a considerable proportion of the sporadic forms of common neurodevelopmental disorders. ${ }^{4,40}$ Note that, as for the other strategies, variants prioritized in this way are more likely to be causative for disease, but de novo occurrence in itself is not sufficient evidence, and follow-up studies are required to identify recurrence or functional proof of pathogenicity. In addition, the focus on variants that are present only in an affected child and not in the parents does not only enrich for de novo mutations but also for sequencing and mapping artifacts, and therefore requires high sequencing accuracy and equal coverage in all three samples investigated. It may therefore be wise to enrich and sequence all samples of a trio in the same experiment. Finally, the de novo strategy requires a relatively high number of three experiments per patient, and will therefore only be applied when none of the other strategies is likely to be successful and parental samples are available.

Candidate strategy. In case of a single dominantly affected individual, without further availability of family members or other affected individuals, the options are limited. Prioritization may be on the basis of predicted impact of the variant on protein function and structure, that is one prioritizes for stop mutations, frame-shifting mutations, and mutations in the canonical splice-sites. In case of missense variants, the two main criteria currently applied are predicted impact on protein structure (eg, Grantham score) and evolutionary conservation of the variant nucleotide (eg, phyloP or GERP scores). Pathogenic mutations tend to have a markedly higher conservation than benign variants. ${ }^{4,41}$ Based on a comparison of scores for benign and pathogenic variants from dbSNP and Human Genome Mutation Database, respectively, we estimated that most pathogenic missense variants have a PhyloP score $>2.5$. $^{4}$ Indeed, so far almost all published missense and nonsense mutations that have been identified by exome sequencing are more conserved than expected (Figure 4).

Similar to the traditional candidate gene approach, information on gene function in relation to the phenotype and what is known or inferred about its pathophysiology may be used in further prioritization. Methods for selecting candidate variants on this scale are relatively new. When there are no obvious candidate variants available (ie, truncating mutations), most studies have turned to computational predictors of the impact of missense variants on the protein structure or function. ${ }^{42}$ There are some practical issues with regard to the utility of these prediction programs for NGS data. Most importantly, however, the sensitivity and specificity needs to be very high, given the large amount of predictions that is performed for a single exome. As an example, one of the two mutations identified as the cause for Miller syndrome by $\mathrm{Ng}$ et $a l^{29}$ was initially missed because it was predicted to be benign. Hence, some studies have used combinations of predictions by different methods to evaluate missense variants. ${ }^{43}$ On the other hand, predictions on a complete set of detected exonic variants will usually yield far too many potential damaging mutations for follow up. ${ }^{44}$ Recently, Erlich et $a l^{45}$ demonstrated how gene prediction tools typically applied in combination with traditional mapping approaches, can also successfully be applied to prioritize candidate genes from exome resequencing experiments. They used three different bioinformatics tools (SUSPECTS, ${ }^{46}$ ToppGene, ${ }^{47}$ and Endeavour ${ }^{15}$ ) to prioritize KIF1A as the most likely candidate gene for hereditary spastic paraparesis. Prioritization of the variants itself using MutationTaster, ${ }^{48}$ Polyphen, ${ }^{49}$ and SIFT $^{50}$ independently pointed to the variant in KIF1A as being the most likely pathogenic variant. This nicely illustrates the potential of combining gene level information with genomic variant information.

In practice, not all studies use a single strategy, and some rely on a combination of approaches. For example Wang et $a l^{51}$ used a combination of a homozygosity and linkage-based approach whereas Musunru et $a l^{52}$ used the combination of a linkage- and double hitbased strategy (Table 1). As a final step in this process, validation by traditional Sanger sequencing as the golden standard for mutation detection is still required. Importantly, definite proof of pathogenicity requires validation in independent patient cohorts and/or functional experiments.

\section{Success rate}

In order to evaluate the success rate of exome sequencing for Mendelian diseases, we investigated the exome coverage of known disease-causing mutations. We considered all 37424 non-synonymous-coding variants annotated as disease-causing in the Human Genome Mutation Database that do not overlap with known dbSNP132 positions. ${ }^{53}$ For each of these mutations, we evaluated the sequence coverage of corresponding targets from 51 exomes, with a median coverage of 49 -fold, that were sequenced in-house using the Agilent $50 \mathrm{Mb}$ exome kit (Agilent Technologies, Inc., Santa Clara, CA, USA) with SOLiD4 sequencing. 
a

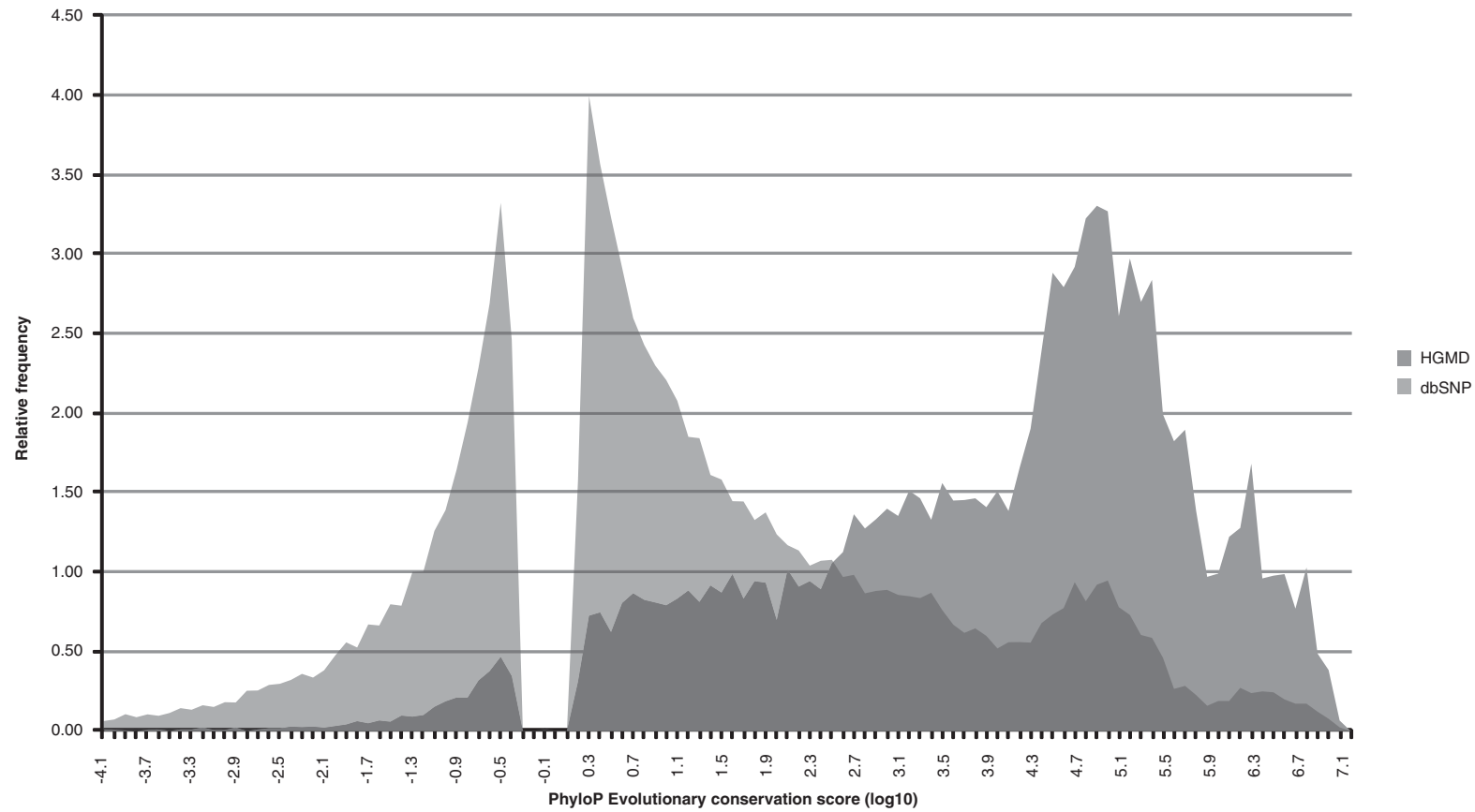

b

PhyloP Evolutionary conservation score $(\log 10)$

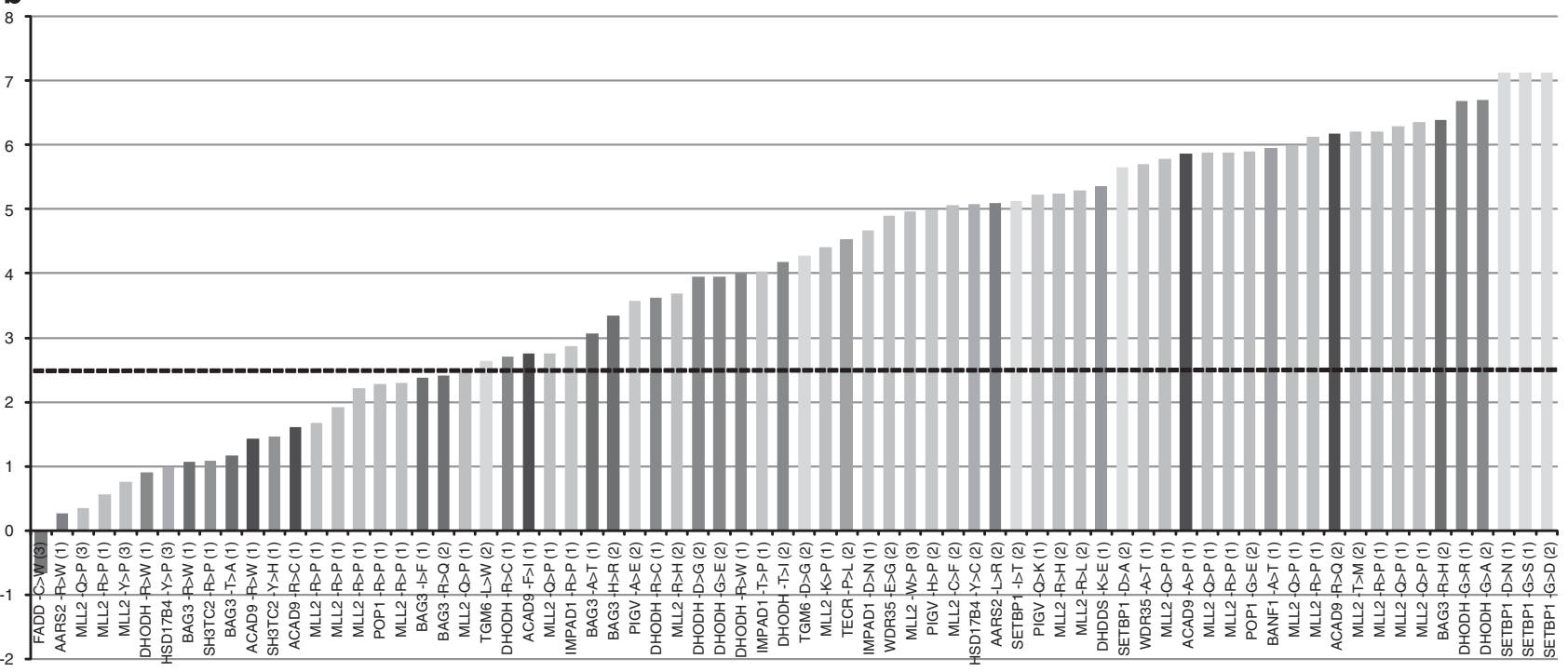

Figure 4 Base pair conservation of published pathogenic missense variants identified by exome sequencing. (a) Histogram plots of PhyloP evolutionary conservation score for non-synonymous variants from dbSNP and Human Genome Mutation Database, showing a clear difference. (b) Conservation of published missense mutations ranked from low to high. Each bar represents the conservation of a missense mutation. Mutations are labeled with gene name, amino-acid change and affected base within the codon (in parentheses). Horizontal dotted line indicates conservation of 2.5 where the distributions of the two distributions from panel (a) intersect. Variants from different studies are indicated by distinct colors. The color reproduction of this figure is available at the European Journal of Human Genetics online.

First, we observed that all 37424 disease-causing variant positions were targeted by the enrichment kit. Of these, 2128 (5.7\%) positions were not covered with sequencing reads at all (median $<1.0$ ), whereas $80.8 \%$ of the positions were covered with a median coverage of at least 10 -fold. Variants that were covered less were typically within regions of either very high or low GC. We conclude that for up to $80 \%$ of all mutations, reliable variants calling would have been possible, that is, a detection with exome sequencing would have been likely.

Additionally, we performed a more biased analysis by evaluating all projects involving unexplained rare Mendelian disorders from the first year of exome sequencing at our institute. We revisited data from 10 likely dominant syndromes as well as 14 suspected recessive disorders.
For 6 out of the 10 dominant diseases, we identified heterozygous mutations in a novel gene. In addition we identified homozygous or compound heterozygous mutations in a novel gene in 8 out of 14 recessive disorders. All these mutations were Sanger-validated and independently replicated in at least one unrelated patient with the same disorder, and/or functionally implicated in the disease. This results in a success rate of $58 \%$ (14 out of 24), a number that may increase slightly as some of the projects are still ongoing.

Among the 10 projects where we did not find any novel genes, in 3 we identified mutations in previously known disease genes $(\sim 13 \%)$. One of the other unsuccessful projects involved Kabuki syndrome, where we sequenced four patients and two unaffected parents, and 
applied both the overlap as well as the de novo approach without identifying the (at that time unknown) causative gene. After the publication of $M L L 2$ as the causative gene for Kabuki syndrome, ${ }^{30}$ we identified the reason for our lack of success. MLL2 was not represented on the exome enrichment kit that we used at that time, and therefore no sequence data for this gene was obtained. While this explains why we were not able to identify any disease-causing mutation, it also shows that we were not misled by false-positive variants and identified wrong candidate genes.

There are several other possible reasons why we are unable to identify the genetic cause by exome sequencing in some projects. Variants have not been identified due to (a) lack of sequence coverage of the variant, (b) bioinformatics variant calling issues, and (c) misinterpretation of variants. Moreover, it may be that (d) the cause of the disease is located outside the coding sequences or (e) being a large indel or structural genomic variant missed by exome sequencing. In addition, for some projects too many candidate variants remained after filtering and no independent recurrence or functional proof has been obtained so far. Finally, clinical heterogeneity or incorrect diagnosis may have falsely impacted our filtering strategy.

\section{CONCLUSION}

The introduction of NGS is transforming Mendelian disease gene identification. No longer is there a need for a complex and timeconsuming laboratory workflow consisting of many different positional cloning approaches. Instead, a single streamlined laboratory workflow will rapidly identify most of the genomic variation present in an individual exome. By applying tailored strategies for disease variant prioritization, many new Mendelian disease genes have been identified in the last 2 years. We estimate that straightforward application of these approaches achieves a success rate of $60-80 \%$ for Mendelian disorders. Improvements in the technology and bioinformatics are likely to increase this success rate. It is therefore likely that exome sequencing will become the most commonly used tool for Mendelian disease gene identification in the next few years.

\section{CONFLICT OF INTEREST}

The authors declare no conflict of interest.

1 McKusick VA Online Mendelian Inheritance in Man, OMIM http://www.ncbi.nIm.nih. gov/omim, http://www.ncbi.nlm.nih.gov/omim, 2011.

2 McClellan J, King MC: Genetic heterogeneity in human disease. Cell 2010; 141: 210-217.

3 Mitchell KJ, Porteous DJ: Rethinking the genetic architecture of schizophrenia. Psychol Med 2011; 41: 19-32.

4 Vissers LE, de Ligt J, Gilissen C et al: A de novo paradigm for mental retardation. Nat Genet 2010; 42: 1109-1112.

$5 \mathrm{Xu} \mathrm{B}$, Roos JL, Dexheimer $\mathrm{P}$ et al: Exome sequencing supports a de novo mutational paradigm for schizophrenia. Nat Genet 2011; 43: 864-868.

6 Antonarakis SE, Beckmann JS: Mendelian disorders deserve more attention. Nat Rev Genet 2006; 7: 277-282.

7 Majewski J, Schwartzentruber J, Lalonde E, Montpetit A, Jabado N: What can exome sequencing do for you? J Med Genet 2011; 48: 580-589.

8 Botstein D, Risch N: Discovering genotypes underlying human phenotypes: past successes for Mendelian disease, future approaches for complex disease. Nat Genet 2003; 33 (Suppl): 228-237.

9 Kurotaki N, Imaizumi K, Harada N et al: Haploinsufficiency of NSD1 causes Sotos syndrome. Nat Genet 2002; 30: 365-366.

10 Kerem B, Rommens JM, Buchanan JA et al: Identification of the cystic fibrosis gene: genetic analysis. Science 1989; 245: 1073-1080.

11 Lander ES, Botstein D: Homozygosity mapping: a way to map human recessive traits with the DNA of inbred children. Science 1987; 236: 1567-1570.

12 Vissers LE, Veltman JA, van Kessel AG, Brunner HG: Identification of disease genes by whole genome CGH arrays. Hum Mol Genet 2005; 14 Spec No. 2: 215.

13 Duerr RH, Taylor KD, Brant SR et al: A genome-wide association study identifies IL23R as an inflammatory bowel disease gene. Science 2006; 314: 1461-1463.
14 Franke $\mathrm{L}$, van $\mathrm{BH}$, Fokkens $\mathrm{L}$, de Jong ED, Egmont-Petersen M, Wijmenga C: Reconstruction of a functional human gene network, with an application for prioritizing positional candidate genes. Am J Hum Genet 2006; 78: 1011-1025.

15 Aerts S, Lambrechts D, Maity S et al: Gene prioritization through genomic data fusion. Nat Biotechnol 2006; 24: 537-544.

16 Tranchevent LC, Capdevila FB, Nitsch D, De MB, De CP, Moreau Y: A guide to web tools to prioritize candidate genes. Brief Bioinform 2011; 12: 22-32.

17 Tiffin N, Andrade-Navarro MA, Perez-Iratxeta C: Linking genes to diseases: it's all in the data. Genome Med 2009; 1: 77.

18 Mardis ER: A decade's perspective on DNA sequencing technology. Nature 2011; 470: 198-203.

19 Ng SB, Nickerson DA, Bamshad MJ, Shendure J: Massively parallel sequencing and rare disease. Hum Mol Genet 2010; 19: 119.

20 Sobreira NL, Cirulli ET, Avramopoulos D et al: Whole-genome sequencing of a single proband together with linkage analysis identifies a Mendelian disease gene. PLoS Genet 2010; 6: 1000991.

21 Lupski JR, Reid JG, Gonzaga-Jauregui C et al: Whole-genome sequencing in a patient with Charcot-Marie-Tooth neuropathy. N Engl J Med 2010; 362: 1181-1191.

22 Roach JC, Glusman G, Smit AF et al: Analysis of genetic inheritance in a family quartet by whole-genome sequencing. Science 2010; 328: 636-639.

23 Ashley EA, Butte AJ, Wheeler MT et al: Clinical assessment incorporating a personal genome. Lancet 2010; 375: 1525-1535.

24 Bainbridge MN, Wiszniewski W, Murdock DR et al: Whole-genome sequencing for optimized patient management. Sci Trans/ Med 2011; 3: 87re3.

25 Mamanova L, Coffey AJ, Scott CE et al: Target-enrichment strategies for nextgeneration sequencing. Nat Methods 2010; 7: 111-118.

26 Durbin RM, Abecasis GR, Altshuler DL et al: A map of human genome variation from population-scale sequencing. Nature 2010; 467: 1061-1073.

$27 \mathrm{NHLBI}$ Exome Sequencing Project (ESP). : Exome Variant Server http://evs.gs. washington.edu/EVS/, 2011.

28 Ng SB, Turner EH, Robertson PD et al: Targeted capture and massively parallel sequencing of 12 human exomes. Nature 2009; 461: 272-276.

$29 \mathrm{Ng} \mathrm{SB}$, Buckingham KJ, Lee $\mathrm{C}$ et al: Exome sequencing identifies the cause of a Mendelian disorder. Nat Genet 2010; 42: 30-35.

$30 \mathrm{Ng} \mathrm{SB}$, Bigham AW, Buckingham KJ et al: Exome sequencing identifies MLL2 mutations as a cause of Kabuki syndrome. Nat Genet 2010; 42: 790-793.

31 Becker J, Semler 0, Gilissen C et al: Exome sequencing identifies truncating mutations in human SERPINF1 in autosomal-recessive osteogenesis imperfecta. Am J Hum Genet 2011; 88: 362-371.

32 Gilissen $\mathrm{C}$, Arts $\mathrm{HH}$, Hoischen A et al: Exome sequencing identifies WDR35 variants involved in Sensenbrenner syndrome. Am J Hum Genet 2010; 87: 418-423.

33 Hoischen A, van Bon BW, Gilissen C et al: De novo mutations of SETBP1 cause Schinzel-Giedion syndrome. Nat Genet 2010; 42: 483-485.

34 Hoischen A, van Bon BW, Rodriguez-Santiago B et al: De novo nonsense mutations in ASXL1 cause Bohring-Opitz syndrome. Nat Genet 2011; 43: 729-731.

35 Walsh T, Shahin H, Elkan-Miller T et al: Whole exome sequencing and homozygosity mapping identify mutation in the cell polarity protein GPSM2 as the cause of nonsyndromic hearing loss DFNB82. Am J Hum Genet 2010; 87: 90-94.

36 Krawitz PM, Schweiger MR, Rodelsperger C et al: Identity-by-descent filtering of exome sequence data identifies PIGV mutations in hyperphosphatasia mental retardation syndrome. Nat Genet 2010; 42: 827-829.

37 Pierce SB, Walsh T, Chisholm KM et al: Mutations in the DBP-deficiency protein HSD17B4 cause ovarian dysgenesis, hearing loss, and ataxia of Perrault Syndrome. Am J Hum Genet 2010; 87: 282-288.

38 Norton N, Li D, Rieder MJ et al: Genome-wide studies of copy number variation and exome sequencing identify rare variants in BAG3 as a cause of dilated cardiomyopathy. Am J Hum Genet 2011; 88: 273-282.

39 O'Roak BJ, Deriziotis P, Lee $\mathrm{C}$ et al: Exome sequencing in sporadic autism spectrum disorders identifies severe de novo mutations. Nat Genet 2011; 43: 585-589.

40 Girard SL, Gauthier J, Noreau A et al: Increased exonic de novo mutation rate in individuals with schizophrenia. Nat Genet 2011; 43: 860-863.

41 Cooper GM, Goode DL, Ng SB et al: Single-nucleotide evolutionary constraint scores highlight disease-causing mutations. Nat Methods 2010; 7: 250-251.

42 Thusberg J, Olatubosun A, Vihinen M: Performance of mutation pathogenicity prediction methods on missense variants. Hum Mutat 2011; 32: 358-368.

43 Shearer AE, DeLuca AP, Hildebrand MS et al: Comprehensive genetic testing for hereditary hearing loss using massively parallel sequencing. Proc Natl Acad Sci USA 2010; 107: 21104-21109.

44 Worthey EA, Mayer AN, Syverson GD et al: Making a definitive diagnosis: successful clinical application of whole exome sequencing in a child with intractable inflammatory bowel disease. Genet Med 2011; 13: 255-262.

45 Erlich Y, Edvardson S, Hodges E et al: Exome sequencing and disease-network analysis of a single family implicate a mutation in KIF1A in hereditary spastic paraparesis. Genome Res 2011; 21: 658-664.

46 Adie EA, Adams RR, Evans KL, Porteous DJ, Pickard BS: SUSPECTS: enabling fast and effective prioritization of positional candidates. Bioinformatics 2006; 22: 773-774.

47 Chen J, Bardes EE, Aronow BJ, Jegga AG: ToppGene Suite for gene list enrichment analysis and candidate gene prioritization. Nucleic Acids Res 2009; 37: W305-W311.

48 Schwarz JM, Rodelsperger C, Schuelke M, Seelow D: MutationTaster evaluates diseasecausing potential of sequence alterations. Nat Methods 2010; 7: 575-576.

49 Adzhubei IA, Schmidt S, Peshkin L et al: A method and server for predicting damaging missense mutations. Nat Methods 2010; 7: 248-249. 
50 Ng PC, Henikoff S: SIFT: predicting amino acid changes that affect protein function. Nucleic Acids Res 2003; 31: 3812-3814.

51 Wang JL, Yang X, Xia K et al: TGM6 identified as a novel causative gene of spinocerebellar ataxias using exome sequencing. Brain 2010; 133: 3510-3518.

52 Musunuru K, Pirruccello JP, Do R et al: Exome sequencing, ANGPTL3 mutations, and familial combined hypolipidemia. N Engl J Med 2010; 363: 2220-2227.

53 Stenson PD, Ball EV, Mort M et al: Human Gene Mutation Database (HGMD): 2003 update. Hum Mutat 2003; 21: 577-581.

54 Johnson JO, Mandrioli J, Benatar M et al: Exome sequencing reveals VCP mutations as a cause of familial ALS. Neuron 2010; 68: 857-864.

55 Zuchner S, Dallman J, Wen R et al: Whole-Exome Sequencing Links a Variant in DHDDS to Retinitis Pigmentosa. Am J Hum Genet 2011; 88: 201-206.

56 Yamaguchi T, Hosomichi K, Narita A et al: Exome resequencing combined with linkage analysis identifies novel PTH1R variants in primary failure of tooth eruption in Japanese. J Bone Miner Res 2011; 26: 1655-1661.

57 Glazov EA, Zankl A, Donskoi M et al: Whole-exome re-sequencing in a family quartet identifies POP1 mutations as the cause of a novel skeletal dysplasia. PLoS Genet 2011; 7: e1002027.

58 Puente XS, Quesada V, Osorio FG et al: Exome sequencing and functional analysis identifies BANF1 mutation as the cause of a hereditary progeroid syndrome. Am J Hum Genet 2011; 88: 650-656.

59 Gunay-Aygun M, Falik-Zaccai TC, Vilboux T et al: NBEAL2 is mutated in gray platelet syndrome and is required for biogenesis of platelet alpha-granules. Nat Genet 2011; 43: 732-734.

60 Weedon MN, Hastings R, Caswell R et al: Exome sequencing identifies a DYNC1H1 mutation in a large pedigree with dominant axonal Charcot-Marie-Tooth disease. Am J Hum Genet 2011; 89: 308-312.

61 Bolze A, Byun M, McDonald D et al: Whole-exome-sequencing-based discovery of human FADD deficiency. Am J Hum Genet 2010; 87: 873-881.

62 Caliskan $\mathrm{M}$, Chong JX, Uricchio $\mathrm{L}$ et al: Exome sequencing reveals a novel mutation for autosomal recessive non-syndromic mental retardation in the TECR gene on chromosome 19p13. Hum Mol Genet 2011; 20: 1285-1289.

63 Bilguvar K, Ozturk AK, Louvi A et al: Whole-exome sequencing identifies recessive WDR62 mutations in severe brain malformations. Nature 2010; 467: 207-210.

64 O'Sullivan J, Bitu CC, Daly SB et al: Whole-exome sequencing identifies FAM20A mutations as a cause of amelogenesis imperfecta and Gingival Hyperplasia syndrome. Am J Hum Genet 2011; 88: 616-620.

65 Barak T, Kwan KY, Louvi A et al: Recessive LAMC3 mutations cause malformations of occipital cortical development. Nat Genet 2011; 43: 590-594.

66 Hanson D, Murray PG, O'Sullivan J et al: Exome sequencing identifies CCDC8 mutations in 3-M syndrome, suggesting that $\mathrm{CCDC} 8$ contributes in a pathway with CUL7 and OBSL1 to control human growth. Am J Hum Genet 2011; 89: 148-153.

67 Shaheen R, Faqeih E, Sunker A et al: Recessive mutations in DOCK6, encoding the guanidine nucleotide exchange factor DOCK6, lead to abnormal actin cytoskeleton organization and Adams-Oliver syndrome. Am J Hum Genet 2011; 89: 328-333.

68 Doi H, Yoshida K, Yasuda T et al: Exome sequencing reveals a homozygous SYT14 mutation in adult-onset, autosomal-recessive spinocerebellar ataxia with psychomotor retardation. Am J Hum Genet 2011; 89: 320-327.
69 Gotz A, Tyynismaa $\mathrm{H}$, Euro $\mathrm{L}$ et al: Exome sequencing identifies mitochondrial alanyltRNA synthetase Mutations in infantile mitochondrial cardiomyopathy. Am J Hum Genet 2011; 88: 635-642.

70 Murdock DR, Clark GD, Bainbridge MN et al: Whole-exome sequencing identifies compound heterozygous mutations in WDR62 in siblings with recurrent polymicrogyria. Am J Med Genet A 2011; 155A: 2071-2077.

$71 \mathrm{Ng} \mathrm{SB}$, Buckingham KJ, Lee $\mathrm{C}$ et al: Exome sequencing identifies the cause of a mendelian disorder. Nat Genet 2010; 42: 30-35.

72 Simpson MA, Irving MD, Asilmaz E et al: Mutations in NOTCH2 cause Hajdu-Cheney syndrome, a disorder of severe and progressive bone loss. Nat Genet 2011; 43 . 303-305.

73 Isidor B, Lindenbaum $\mathrm{P}$, Pichon $\mathrm{O}$ et al: Truncating mutations in the last exon of NOTCH2 cause a rare skeletal disorder with osteoporosis. Nat Genet 2011; 43: 306-308.

74 Vissers LE, Lausch E, Unger S et al: Chondrodysplasia and abnormal joint development associated with mutations in IMPAD1, encoding the golgi-resident nucleotide phosphatase, gPAPP. Am J Hum Genet 2011; 88: 608-615.

75 Albers CA, Cvejic A, Favier R et al: Exome sequencing identifies NBEAL2 as the causative gene for gray platelet syndrome. Nat Genet 2011; 43: 735-737.

76 Dickinson RE, Griffin H, Bigley V et al: Exome sequencing identifies GATA-2 mutation as the cause of dendritic cell, monocyte, B and NK lymphoid deficiency. Blood 2011; 118: 2656-2658.

77 Sirmaci A, Spiliopoulos M, Brancati F et al: Mutations in ANKRD11 cause KBG Syndrome, characterized by intellectual disability, skeletal malformations, and macrodontia. Am J Hum Genet 2011; 89: 289-294.

78 Agrawal N, Frederick MJ, Pickering CR et al: Exome sequencing of head and neck squamous cell carcinoma reveals inactivating mutations in NOTCH1. Science 2011; 333: 1154-1157.

79 Byun M, Abhyankar A, Lelarge $V$ et al: Whole-exome sequencing-based discovery of STIM1 deficiency in a child with fatal classic Kaposi sarcoma. J Exp Med 2010; 207: 2307-2312.

80 Haack TB, Danhauser K, Haberberger B et al: Exome sequencing identifies ACAD9 mutations as a cause of complex I deficiency. Nat Genet 2010; 42: 1131-1134.

81 Ozgul RK, Siemiatkowska AM, Yucel D et al: Exome sequencing and cis-regulatory mapping identify mutations in MAK, a gene encoding a regulator of ciliary length, as a cause of retinitis pigmentosa. Am J Hum Genet 2011; 89: 253-264.

82 Klein CJ, Botuyan MV, Wu Y et al: Mutations in DNMT1 cause hereditary sensory neuropathy with dementia and hearing loss. Nat Genet 2011; 43: 595-600.

83 Puente XS, Quesada V, Osorio FG et al: Exome sequencing and functional analysis identifies BANF1 mutation as the cause of a Hereditary Progeroid syndrome. Am J Hum Genet 2011; 88: 650-656.

84 Kalay E, Yigit G, Aslan Y et al: CEP152 is a genome maintenance protein disrupted in Seckel syndrome. Nat Genet 2011; 43: 23-26.

85 O'Sullivan J, Bitu CC, Daly SB et al: Whole-exome sequencing identifies FAM20A mutations as a cause of Amelogenesis imperfecta and Gingival Hyperplasia syndrome. Am J Hum Genet 2011; 88: 616-620. 